\title{
Description of the Magnetic Field and Divergence of Multisolenoid Aharonov-Bohm Potential
}

\author{
Araz R. Aliev, ${ }^{1,2,3}$ Elshad H. Eyvazov, ${ }^{2,3}$ Said F. M. Ibrahim, ${ }^{4}$ and Hassan A. Zedan ${ }^{5}$ \\ ${ }^{1}$ Azerbaijan State University of Oil and Industry, 1010 Baku, Azerbaijan \\ ${ }^{2}$ Baku State University, 1148 Baku, Azerbaijan \\ ${ }^{3}$ Institute of Mathematics and Mechanics of ANAS, 1141 Baku, Azerbaijan \\ ${ }^{4} J e d d a h$ University, Jeddah 21895, Saudi Arabia \\ ${ }^{5}$ Kafr El-Sheikh University, Kafr El-Sheikh 33516, Egypt \\ Correspondence should be addressed to Araz R. Aliev; alievaraz@yahoo.com
}

Received 1 September 2015; Accepted 11 January 2016

Academic Editor: Emilio Elizalde

Copyright (C) 2016 Araz R. Aliev et al. This is an open access article distributed under the Creative Commons Attribution License, which permits unrestricted use, distribution, and reproduction in any medium, provided the original work is properly cited.

\begin{abstract}
Explicit formulas for the magnetic field and divergence of multisolenoid Aharonov-Bohm potential are obtained; the mathematical essence of this potential is explained. It is shown that the magnetic field and divergence of this potential are very singular generalized functions concentrated at a finite number of thin solenoids. Deficiency index is found for the minimal operator generated by the Aharonov-Bohm differential expression.
\end{abstract}

\section{Introduction}

66 years have passed since the publication of Aharonov and Bohm's "Significance of Electromagnetic Potential in the Quantum Theory" [1], and since then interest in this paper has never faded. According to Web of Science-Google Scholar, it has been cited 5680 times (as of December 2014)! Note that there are plenty of both supporters and opponents of this work (see, e.g., $[2,3])$.

The purpose of our work is to find explicit formulas for the magnetic field and divergence of multisolenoid Aharonov-Bohm potential and to explain the mathematical essence of it. The obtained formulas show (see Theorems 1 and 3) that the magnetic field and divergence of this potential are very singular generalized functions concentrated at a finite number of thin solenoids perpendicular to the plane $x_{1} O x_{2}$.

\section{Main Results}

Let $\xi_{k}=\left(x_{1}^{(k)}, x_{2}^{(k)}\right), k=1,2, \ldots, n$, be pairwise distinct points in $R_{2}$, let $a_{k}: S_{1}(0) \rightarrow R_{1}, k=1,2, \ldots, n$, be real, bounded, and measurable functions on the unit circle $S_{1}(0) \subset R_{2}$, and $\Omega^{\prime}=R_{2} \backslash\left\{\xi_{k}, k=1,2, \ldots, n\right\}$. Define the magnetic Aharonov-Bohm potential as follows:

$$
\begin{array}{r}
A(x) \\
=\sum_{k=1}^{n} a_{k}\left(\frac{x-\xi_{k}}{\left|x-\xi_{k}\right|}\right) \frac{1}{\left|x-\xi_{k}\right|^{2}}\left(-x_{2}+x_{2}^{(k)}, x_{1}-x_{1}^{(k)}\right), \\
x=\left(x_{1}, x_{2}\right) \in \Omega^{\prime},
\end{array}
$$

where

$$
\left|x-\xi_{k}\right|=\sqrt{\left(x_{1}-x_{1}^{(k)}\right)^{2}+\left(x_{2}-x_{2}^{(k)}\right)^{2}} .
$$

As far as we know, in all the earlier works (except for [4]) dedicated to the Aharonov-Bohm effect (for short, AB effect), the functions $a_{k}\left(\left(x-\xi_{k}\right) /\left|x-\xi_{k}\right|\right), k=1,2, \ldots, n$, are constants.

The following theorems are true (in case $n=1$ they were proved in [4]). 
Theorem 1. Let the magnetic field $B=\nabla \times A$ be generated by the magnetic Aharonov-Bohm potential (1) in the sense of generalized functions. Then the following equality is true:

$$
B=\nabla \times A=\sum_{k=1}^{n}\left[\int_{-\pi}^{\pi} a_{k}(\theta) d \theta\right] \delta\left(x-\xi_{k}\right),
$$

where $\delta\left(x-\xi_{k}\right), k=1,2, \ldots, n$, are the Dirac functions and $\nabla=\left(\partial / \partial x_{1}, \partial / \partial x_{2}\right)$ is the gradient operator.

Proof. Let

$$
A(x)=\left(A_{x_{1}}, A_{x_{2}}\right)
$$

where

$$
\begin{aligned}
& A_{x_{1}}=\sum_{k=1}^{n} a_{k}\left(\frac{x-\xi_{k}}{\left|x-\xi_{k}\right|}\right) \frac{-x_{2}+x_{2}^{(k)}}{\left|x-\xi_{k}\right|^{2}}, \\
& A_{x_{2}}=\sum_{k=1}^{n} a_{k}\left(\frac{x-\xi_{k}}{\left|x-\xi_{k}\right|}\right) \frac{x_{1}-x_{1}^{(k)}}{\left|x-\xi_{k}\right|^{2}} .
\end{aligned}
$$

Then the definition of magnetic field

$$
B=\nabla \times A=\frac{\partial A_{x_{2}}}{\partial x_{1}}-\frac{\partial A_{x_{1}}}{\partial x_{2}}
$$

$$
\begin{array}{rl}
\int_{R_{2}} & B f(x) d x \\
= & \int_{R_{2}}\left\{\left[\sum_{k=1}^{n} a_{k}\left(\frac{x-\xi_{k}}{\left|x-\xi_{k}\right|}\right) \frac{-x_{2}+x_{2}^{(k)}}{\left|x-\xi_{k}\right|^{2}}\right] \frac{\partial f\left(x_{1}, x_{2}\right)}{\partial x_{2}}-\left[\sum_{k=1}^{n} a_{k}\left(\frac{x-\xi_{k}}{\left|x-\xi_{k}\right|}\right) \frac{x_{1}-x_{1}^{(k)}}{\left|x-\xi_{k}\right|^{2}}\right] \frac{\partial f\left(x_{1}, x_{2}\right)}{\partial x_{1}}\right\} d x_{1} d x_{2} \\
= & \sum_{k=1}^{n}\left\{\int_{R_{2}} a_{k}\left(\frac{x-\xi_{k}}{\left|x-\xi_{k}\right|}\right)\left[\frac{-x_{2}+x_{2}^{(k)}}{\left|x-\xi_{k}\right|^{2}} \frac{\partial f\left(x_{1}, x_{2}\right)}{\partial x_{2}}-\frac{x_{1}-x_{1}^{(k)}}{\left|x-\xi_{k}\right|^{2}} \frac{\partial f\left(x_{1}, x_{2}\right)}{\partial x_{1}}\right]\right\} d x_{1} d x_{2}=-\sum_{k=1}^{n} J_{k}(f),
\end{array}
$$

where

$$
\begin{array}{r}
J_{k}(f)=\int_{R_{2}} a_{k}\left(\frac{x-\xi_{k}}{\left|x-\xi_{k}\right|}\right)\left[\frac{x_{1}-x_{1}^{(k)}}{\left|x-\xi_{k}\right|^{2}} \frac{\partial f\left(x_{1}, x_{2}\right)}{\partial x_{1}}\right. \\
\left.+\frac{x_{2}-x_{2}^{(k)}}{\left|x-\xi_{k}\right|^{2}} \frac{\partial f\left(x_{1}, x_{2}\right)}{\partial x_{2}}\right] d x_{1} d x_{2}, \\
k=1,2, \ldots, n .
\end{array}
$$

Using the transformation of plane into itself defined by the formulas

$$
\begin{aligned}
& t_{1}=x_{1}-x_{1}^{(k)}, \\
& t_{2}=x_{2}-x_{2}^{(k)},
\end{aligned}
$$

implies that for every function $f(x) \in C_{0}^{\infty}\left(R_{2}\right)$ we have

$$
\begin{array}{rl}
\int_{R_{2}} & B f(x) d x \\
= & \int_{R_{2}}\left(\frac{\partial A_{x_{2}}}{\partial x_{1}}-\frac{\partial A_{x_{1}}}{\partial x_{2}}\right) f\left(x_{1}, x_{2}\right) d x_{1} d x_{2} .
\end{array}
$$

Taking into account the identity

$$
\begin{aligned}
& \left(\frac{\partial A_{x_{2}}}{\partial x_{1}}-\frac{\partial A_{x_{1}}}{\partial x_{2}}\right) f\left(x_{1}, x_{2}\right) \\
& \quad=\frac{\partial}{\partial x_{1}}\left(A_{x_{2}} f\right)-\frac{\partial}{\partial x_{2}}\left(A_{x_{1}} f\right)-A_{x_{2}} \frac{\partial f}{\partial x_{1}}+A_{x_{1}} \frac{\partial f}{\partial x_{2}}
\end{aligned}
$$

and the Green formula, we rewrite relation (7) as follows:

$$
\begin{array}{rl}
\int_{R_{2}} & B f(x) d x \\
\quad= & \int_{R_{2}}\left(A_{x_{1}} \frac{\partial f\left(x_{1}, x_{2}\right)}{\partial x_{2}}-A_{x_{2}} \frac{\partial f\left(x_{1}, x_{2}\right)}{\partial x_{1}}\right) d x_{1} d x_{2} .
\end{array}
$$

Hence, by virtue of (5), we get and considering the equalities

$$
\begin{aligned}
& \frac{\partial f\left(x_{1}, x_{2}\right)}{\partial x_{1}}=\frac{\partial f\left(t_{1}+x_{1}^{(k)}, t_{2}+x_{2}^{(k)}\right)}{\partial t_{1}} \\
& \frac{\partial f\left(x_{1}, x_{2}\right)}{\partial x_{2}}=\frac{\partial f\left(t_{1}+x_{1}^{(k)}, t_{2}+x_{2}^{(k)}\right)}{\partial t_{2}}
\end{aligned}
$$

in (11), we arrive at the following formula:

$$
\begin{gathered}
J_{k}(f)=\int_{R_{2}} a_{k}\left(\frac{t}{|t|}\right)\left[\frac{t_{1}}{|t|^{2}} \frac{\partial f\left(t_{1}+x_{1}^{(k)}, t_{2}+x_{2}^{(k)}\right)}{\partial t_{1}}\right. \\
\left.+\frac{t_{2}}{|t|^{2}} \frac{\partial f\left(t_{1}+x_{1}^{(k)}, t_{2}+x_{2}^{(k)}\right)}{\partial t_{2}}\right] d t_{1} d t_{2},
\end{gathered}
$$


After transition to polar coordinates

$$
\begin{aligned}
& t_{1}=r \cos \theta, \\
& t_{2}=r \sin \theta,
\end{aligned}
$$

$$
r>0, \quad-\pi<\theta \leq \pi(t=r(\cos \theta, \sin \theta)),
$$

and using the equalities

$$
\begin{gathered}
\frac{\partial f\left(t_{1}+x_{1}^{(k)}, t_{2}+x_{2}^{(k)}\right)}{\partial t_{1}} \\
=\frac{\partial f\left(r \cos \theta+x_{1}^{(k)}, r \sin \theta+x_{2}^{(k)}\right)}{\partial r} \frac{\partial r}{\partial t_{1}} \\
\quad+\frac{\partial f\left(r \cos \theta+x_{1}^{(k)}, r \sin \theta+x_{2}^{(k)}\right)}{\partial \theta} \frac{\partial \theta}{\partial t_{1}} \\
=\frac{\partial f\left(r \cos \theta+x_{1}^{(k)}, r \sin \theta+x_{2}^{(k)}\right)}{\partial r} \cos \theta
\end{gathered}
$$

$$
\begin{aligned}
J_{k}(f) & =\int_{0}^{+\infty} \int_{-\pi}^{\pi} a_{k}(\cos \theta, \sin \theta)\{\cos \theta \\
& \cdot\left[\frac{\partial f\left(r \cos \theta+x_{1}^{(k)}, r \sin \theta+x_{2}^{(k)}\right)}{\partial r} \cos \theta-\frac{\partial f\left(r \cos \theta+x_{1}^{(k)}, r \sin \theta+x_{2}^{(k)}\right)}{\partial \theta} \frac{\sin \theta}{r}\right]+\sin \theta \\
& \left.\cdot\left[\frac{\partial f\left(r \cos \theta+x_{1}^{(k)}, r \sin \theta+x_{2}^{(k)}\right)}{\partial r} \sin \theta+\frac{\partial f\left(r \cos \theta+x_{1}^{(k)}, r \sin \theta+x_{2}^{(k)}\right)}{\partial \theta} \frac{\cos \theta}{r}\right]\right\} d r d \theta \\
& =\int_{0}^{+\infty} \int_{-\pi}^{\pi} a_{k}(\cos \theta, \sin \theta) \frac{\partial f\left(r \cos \theta+x_{1}^{(k)}, r \sin \theta+x_{2}^{(k)}\right)}{\partial r} d r d \theta .
\end{aligned}
$$

Taking into account $f(x) \in C_{0}^{\infty}\left(R_{2}\right)$ and denoting $a_{k}(\theta) \equiv$ $a_{k}(\cos \theta, \sin \theta)$, from (17) we have

$$
\begin{aligned}
J_{k}(f) & =-f\left(x_{1}^{(k)}, x_{2}^{(k)}\right) \int_{-\pi}^{\pi} a_{k}(\theta) d \theta \\
& =-f\left(\xi_{k}\right) \int_{-\pi}^{\pi} a_{k}(\theta) d \theta .
\end{aligned}
$$

The Dirac function $\delta\left(x-\xi_{k}\right)$ acts as follows:

$$
\left(\delta\left(x-\xi_{k}\right), f(x)\right)=f\left(\xi_{k}\right) .
$$

Then the functional defined by the right-hand side of (18) is a generalized function. Thus, formula (18) can be rewritten in the following way:

$$
\begin{array}{r}
J_{k}(f)=-\left[\int_{-\pi}^{\pi} a_{k}(\theta) d \theta\right]\left(\delta\left(x-\xi_{k}\right), f(x)\right) \\
=-\left(\left[\int_{-\pi}^{\pi} a_{k}(\theta) d \theta\right] \delta\left(x-\xi_{k}\right), f(x)\right), \\
k=1,2, \ldots, n .
\end{array}
$$

Due to (20), equality (10) has the following form:

$$
\begin{aligned}
(B, f(x)) & \equiv \int_{R_{2}} B f(x) d x \\
& =\sum_{k=1}^{n}\left(\left[\int_{-\pi}^{\pi} a_{k}(\theta) d \theta\right] \delta\left(x-\xi_{k}\right), f(x)\right) \\
& =\left(\sum_{k=1}^{n}\left[\int_{-\pi}^{\pi} a_{k}(\theta) d \theta\right] \delta\left(x-\xi_{k}\right), f(x)\right) .
\end{aligned}
$$

Consequently, we have

$$
B=\nabla \times A=\sum_{k=1}^{n}\left[\int_{-\pi}^{\pi} a_{k}(\theta) d \theta\right] \delta\left(x-\xi_{k}\right) .
$$

The theorem is proved.

Remark 2. The formula

$$
B=\sum_{k=1}^{n}\left[\int_{-\pi}^{\pi} a_{k}(\theta) d \theta\right] \delta\left(x-\xi_{k}\right)
$$


implies that if the condition

$$
\int_{-\pi}^{\pi} a_{k}(\theta) d \theta=0
$$

holds for every $k$ from $\{1,2, \ldots, n\}$, then the $\mathrm{AB}$ effect is absent because the total magnetic flux of the magnetic field $B$ passing through the closed contour that covers all the points $\xi_{k}=\left(x_{1}^{(k)}, x_{2}^{(k)}\right), k=1,2, \ldots, n$, is equal to zero.

The conditions for both the presence and absence of the $\mathrm{AB}$ effect in multiply connected domains are thoroughly studied in $[3,5]$.

Theorem 3. Let the divergence $\operatorname{div} A=\nabla \cdot A$ be generated by the magnetic Aharonov-Bohm potential (1) in the sense of generalized functions. Then the following equality is true:

$$
\begin{aligned}
\operatorname{div} A & =\sum_{k=1}^{n} V \cdot p \cdot\left\{\frac { 1 } { | x - \xi _ { k } | ^ { 2 } } \left[-\frac{\partial a_{k}\left(\left(x_{1}-x_{1}^{(k)}\right) /\left|x-\xi_{k}\right|,\left(x_{2}-x_{2}^{(k)}\right) /\left|x-\xi_{k}\right|\right)}{\partial\left(\left(x_{1}-x_{1}^{(k)}\right) /\left|x-\xi_{k}\right|\right)} \frac{x_{2}-x_{2}^{(k)}}{\left|x-\xi_{k}\right|}\right.\right. \\
+ & \left.\left.\frac{\partial a_{k}\left(\left(x_{1}-x_{1}^{(k)}\right) /\left|x-\xi_{k}\right|,\left(x_{2}-x_{2}^{(k)}\right) /\left|x-\xi_{k}\right|\right)}{\partial\left(\left(x_{2}-x_{2}^{(k)}\right) /\left|x-\xi_{k}\right|\right)} \frac{x_{1}-x_{1}^{(k)}}{\left|x-\xi_{k}\right|}\right]\right\}
\end{aligned}
$$

where

$$
\begin{aligned}
V . p . & \left\{\frac { 1 } { | x - \xi _ { k } | ^ { 2 } } \left[-\frac{\partial a_{k}\left(\left(x_{1}-x_{1}^{(k)}\right) /\left|x-\xi_{k}\right|,\left(x_{2}-x_{2}^{(k)}\right) /\left|x-\xi_{k}\right|\right)}{\partial\left(\left(x_{1}-x_{1}^{(k)}\right) /\left|x-\xi_{k}\right|\right)} \frac{x_{2}-x_{2}^{(k)}}{\left|x-\xi_{k}\right|}\right.\right. \\
+ & \left.\left.\frac{\partial a_{k}\left(\left(x_{1}-x_{1}^{(k)}\right) /\left|x-\xi_{k}\right|,\left(x_{2}-x_{2}^{(k)}\right) /\left|x-\xi_{k}\right|\right)}{\partial\left(\left(x_{2}-x_{2}^{(k)}\right) /\left|x-\xi_{k}\right|\right)} \frac{x_{1}-x_{1}^{(k)}}{\left|x-\xi_{k}\right|}\right]\right\}, \quad k=1,2, \ldots, n,
\end{aligned}
$$

are singular generalized functions; the letters V.p. mean "Cauchy principal value."
Proof. Let $f(x) \in C_{0}^{\infty}\left(R_{2}\right)$. Then, by the definition of the derivative of generalized function, using formulas (5), we have

$$
\begin{aligned}
(\operatorname{div} A, f(x)) & =(\nabla \cdot A, f(x))=\int_{R_{2}}\left(\frac{\partial A_{x_{1}}}{\partial x_{1}}+\frac{\partial A_{x_{2}}}{\partial x_{2}}\right) f(x) d x=-\int_{R_{2}}\left(A_{x_{1}} \frac{\partial f(x)}{\partial x_{1}}+A_{x_{2}} \frac{\partial f(x)}{\partial x_{2}}\right) d x \\
& =-\sum_{k=1}^{n}\left\{\lim _{0<\delta \rightarrow 0} \int_{\left|x-\xi_{k}\right| \geq \delta} a_{k}\left(\frac{x-\xi_{k}}{\left|x-\xi_{k}\right|}\right)\left[\frac{-x_{2}+x_{2}^{(k)}}{\left|x-\xi_{k}\right|^{2}} \frac{\partial f\left(x_{1}, x_{2}\right)}{\partial x_{1}}+\frac{x_{1}-x_{1}^{(k)}}{\left|x-\xi_{k}\right|^{2}} \frac{\partial f\left(x_{1}, x_{2}\right)}{\partial x_{2}}\right] d x_{1} d x_{2}\right\} \\
& =-\sum_{k=1}^{n}\left[\lim _{0<\delta \rightarrow 0} I_{k, \delta}(f)\right]
\end{aligned}
$$

where

$$
\begin{gathered}
I_{k, \delta}(f)=\int_{\left|x-\xi_{k}\right| \geq \delta} a_{k}\left(\frac{x-\xi_{k}}{\left|x-\xi_{k}\right|}\right) \\
\cdot\left[\frac{-x_{2}+x_{2}^{(k)}}{\left|x-\xi_{k}\right|^{2}} \frac{\partial f\left(x_{1}, x_{2}\right)}{\partial x_{1}}\right.
\end{gathered}
$$$$
\left.+\frac{x_{1}-x_{1}^{(k)}}{\left|x-\xi_{k}\right|^{2}} \frac{\partial f\left(x_{1}, x_{2}\right)}{\partial x_{2}}\right] d x_{1} d x_{2}
$$$$
k=1,2, \ldots, n \text {. }
$$

Using substitutions (12) and (15) and formulas (13) and (16), we obtain 


$$
\begin{aligned}
& I_{k, \delta}(f)=\int_{|t| \geq \delta} a_{k}\left(\frac{t}{|t|}\right)\left[\frac{-t_{2}}{|t|^{2}} \frac{\partial f\left(t_{1}+x_{1}^{(k)}, t_{2}+x_{2}^{(k)}\right)}{\partial t_{1}}+\frac{t_{1}}{|t|^{2}} \frac{\partial f\left(t_{1}+x_{1}^{(k)}, t_{2}+x_{2}^{(k)}\right)}{\partial t_{2}}\right] d t_{1} d t_{2}=\int_{\delta}^{+\infty} \int_{-\pi}^{\pi} a_{k}(\cos \theta, \sin \theta) \\
& \quad \cdot\left\{-\sin \theta\left[\frac{\partial f\left(r \cos \theta+x_{1}^{(k)}, r \sin \theta+x_{2}^{(k)}\right)}{\partial r} \cos \theta-\frac{\partial f\left(r \cos \theta+x_{1}^{(k)}, r \sin \theta+x_{2}^{(k)}\right)}{\partial \theta} \frac{\sin \theta}{r}\right]+\cos \theta\right. \\
& \left.\quad \cdot\left[\frac{\partial f\left(r \cos \theta+x_{1}^{(k)}, r \sin \theta+x_{2}^{(k)}\right)}{\partial r} \sin \theta+\frac{\partial f\left(r \cos \theta+x_{1}^{(k)}, r \sin \theta+x_{2}^{(k)}\right)}{\partial \theta} \frac{\cos \theta}{r}\right]\right\} d r d \theta \\
& \quad=\int_{\delta}^{+\infty} \int_{-\pi}^{\pi} a_{k}(\theta) \frac{\partial f\left(r \cos \theta+x_{1}^{(k)}, r \sin \theta+x_{2}^{(k)}\right)}{\partial \theta} \frac{1}{r} d r d \theta=\int_{\delta}^{+\infty} \frac{1}{r} d r \int_{-\pi}^{\pi} a_{k}(\theta) \frac{\partial f\left(r \cos \theta+x_{1}^{(k)}, r \sin \theta+x_{2}^{(k)}\right)}{\partial \theta} d \theta \\
& \quad=-\int_{\delta}^{+\infty} \frac{1}{r} d r \int_{-\pi}^{\pi} a_{k}^{\prime}(\theta) f\left(r \cos \theta+x_{1}^{(k)}, r \sin \theta+x_{2}^{(k)}\right) d \theta=\int_{\delta}^{+\infty} \frac{1}{r} d r \int_{-\pi}^{\pi} a_{k}(\theta) \frac{\partial f\left(r \cos \theta+x_{1}^{(k)}, r \sin \theta+x_{2}^{(k)}\right)}{\partial \theta} d \theta, \\
& k=1,2, \ldots, n .
\end{aligned}
$$

Now, to express $a_{k}^{\prime}(\theta)$ in Cartesian coordinates $x_{1}$ and $x_{2}$, we put

$$
\begin{aligned}
& M_{k}\left(x_{1}, x_{2}\right) \equiv a_{k}\left(\frac{x_{1}-x_{1}^{(k)}}{\left|x-\xi_{k}\right|}, \frac{x_{2}-x_{2}^{(k)}}{\left|x-\xi_{k}\right|}\right) \\
&=a_{k}(\cos \theta, \sin \theta) \equiv a_{k}(\theta), \\
& \quad k=1,2, \ldots, n .
\end{aligned}
$$

we find

$$
\begin{aligned}
a_{k}^{\prime}(\theta)= & \frac{\partial M_{k}}{\partial \theta}=\frac{\left|\begin{array}{l}
\cos \theta \partial M_{k}\left(x_{1}, x_{2}\right) / \partial x_{1} \\
\sin \theta \partial M_{k}\left(x_{1}, x_{2}\right) / \partial x_{2}
\end{array}\right|}{\left|\begin{array}{l}
\cos \theta-\sin \theta / r \\
\sin \theta \cos \theta / r
\end{array}\right|} \\
= & r\left(\frac{\partial M_{k}\left(x_{1}, x_{2}\right)}{\partial x_{2}} \cos \theta-\frac{\partial M_{k}\left(x_{1}, x_{2}\right)}{\partial x_{1}} \sin \theta\right) \\
= & \frac{\partial M_{k}\left(x_{1}, x_{2}\right)}{\partial x_{2}}\left(x_{1}-x_{1}^{(k)}\right) \\
& -\frac{\partial M_{k}\left(x_{1}, x_{2}\right)}{\partial x_{1}}\left(x_{2}-x_{2}^{(k)}\right), \quad k=1,2, \ldots, n .
\end{aligned}
$$

Differentiating the composite function $M_{k}\left(x_{1}, x_{2}\right)$ in $x_{1}$ and $x_{2}$ and using formula (32), we find

$$
\begin{aligned}
& a_{k}^{\prime}(\theta)=\left[\frac{\partial a_{k}\left(\left(x_{1}-x_{1}^{(k)}\right) /\left|x-\xi_{k}\right|,\left(x_{2}-x_{2}^{(k)}\right) /\left|x-\xi_{k}\right|\right)}{\partial\left(\left(x_{1}-x_{1}^{(k)}\right) /\left|x-\xi_{k}\right|\right)}\left(\frac{-\left(x_{1}-x_{1}^{(k)}\right)\left(x_{2}-x_{2}^{(k)}\right)}{\left|x-\xi_{k}\right|^{3}}\right)\right. \\
& \left.+\frac{\partial a_{k}\left(\left(x_{1}-x_{1}^{(k)}\right) /\left|x-\xi_{k}\right|,\left(x_{2}-x_{2}^{(k)}\right) /\left|x-\xi_{k}\right|\right)}{\partial\left(\left(x_{2}-x_{2}^{(k)}\right) /\left|x-\xi_{k}\right|\right)}\left(\frac{1}{\left|x-\xi_{k}\right|}-\frac{\left(x_{2}-x_{2}^{(k)}\right)^{2}}{\left|x-\xi_{k}\right|^{3}}\right)\right]\left(x_{1}-x_{1}^{(k)}\right) \\
& +\left[\frac{\partial a_{k}\left(\left(x_{1}-x_{1}^{(k)}\right) /\left|x-\xi_{k}\right|,\left(x_{2}-x_{2}^{(k)}\right) /\left|x-\xi_{k}\right|\right)}{\partial\left(\left(x_{1}-x_{1}^{(k)}\right) /\left|x-\xi_{k}\right|\right)}\left(\frac{1}{\left|x-\xi_{k}\right|}-\frac{\left(x_{1}-x_{1}^{(k)}\right)^{2}}{\left|x-\xi_{k}\right|^{3}}\right)\right. \\
& \left.+\frac{\partial a_{k}\left(\left(x_{1}-x_{1}^{(k)}\right) /\left|x-\xi_{k}\right|,\left(x_{2}-x_{2}^{(k)}\right) /\left|x-\xi_{k}\right|\right)}{\partial\left(\left(x_{2}-x_{2}^{(k)}\right) /\left|x-\xi_{k}\right|\right)}\left(\frac{-\left(x_{1}-x_{1}^{(k)}\right)\left(x_{2}-x_{2}^{(k)}\right)}{\left|x-\xi_{k}\right|^{3}}\right)\right]\left(x_{2}-x_{2}^{(k)}\right)
\end{aligned}
$$




$$
\begin{aligned}
& =-\frac{\partial a_{k}\left(\left(x_{1}-x_{1}^{(k)}\right) /\left|x-\xi_{k}\right|,\left(x_{2}-x_{2}^{(k)}\right) /\left|x-\xi_{k}\right|\right)}{\partial\left(\left(x_{1}-x_{1}^{(k)}\right) /\left|x-\xi_{k}\right|\right)} \frac{x_{2}-x_{2}^{(k)}}{\left|x-\xi_{k}\right|}+\frac{\partial a_{k}\left(\left(x_{1}-x_{1}^{(k)}\right) /\left|x-\xi_{k}\right|,\left(x_{2}-x_{2}^{(k)}\right) /\left|x-\xi_{k}\right|\right)}{\partial\left(\left(x_{2}-x_{2}^{(k)}\right) /\left|x-\xi_{k}\right|\right)} \\
& \cdot \frac{x_{1}-x_{1}^{(k)}}{\left|x-\xi_{k}\right|}, \quad k=1,2, \ldots, n .
\end{aligned}
$$

Passing to the limit in (29) as $\delta \rightarrow 0$ and taking into account

(33), we obtain

$$
\begin{gathered}
\lim _{0<\delta \rightarrow 0} I_{k, \delta}(f)=-\mathrm{V} \cdot \mathrm{p} \cdot \int_{R_{2}}\left\{\frac { 1 } { | x - \xi _ { k } | ^ { 2 } } \left[-\frac{\partial a_{k}\left(\left(x_{1}-x_{1}^{(k)}\right) /\left|x-\xi_{k}\right|,\left(x_{2}-x_{2}^{(k)}\right) /\left|x-\xi_{k}\right|\right)}{\partial\left(\left(x_{1}-x_{1}^{(k)}\right) /\left|x-\xi_{k}\right|\right)} \frac{x_{2}-x_{2}^{(k)}}{\left|x-\xi_{k}\right|}\right.\right. \\
\left.\left.+\frac{\partial a_{k}\left(\left(x_{1}-x_{1}^{(k)}\right) /\left|x-\xi_{k}\right|,\left(x_{2}-x_{2}^{(k)}\right) /\left|x-\xi_{k}\right|\right)}{\partial\left(\left(x_{2}-x_{2}^{(k)}\right) /\left|x-\xi_{k}\right|\right)} \frac{x_{1}-x_{1}^{(k)}}{\left|x-\xi_{k}\right|}\right]\right\} f(x) d x, \quad k=1,2, \ldots, n .
\end{gathered}
$$

It is seen from (27) and (34) that the following equality is true for every $f(x) \in C_{0}^{\infty}\left(R_{2}\right)$ :

$(\operatorname{div} A, f(x))$

$$
\begin{aligned}
& =\sum_{k=1}^{n} \mathrm{~V} \cdot \mathrm{p} \cdot \int_{R_{2}}\left\{\frac{1}{\left|x-\xi_{k}\right|^{2}}\left[-\frac{\partial a_{k}\left(\left(x_{1}-x_{1}^{(k)}\right) /\left|x-\xi_{k}\right|,\left(x_{2}-x_{2}^{(k)}\right) /\left|x-\xi_{k}\right|\right)}{\partial\left(\left(x_{1}-x_{1}^{(k)}\right) /\left|x-\xi_{k}\right|\right)} \frac{x_{2}-x_{2}^{(k)}}{\left|x-\xi_{k}\right|}+\frac{\partial a_{k}\left(\left(x_{1}-x_{1}^{(k)}\right) /\left|x-\xi_{k}\right|,\left(x_{2}-x_{2}^{(k)}\right) /\left|x-\xi_{k}\right|\right)}{\partial\left(\left(x_{2}-x_{2}^{(k)}\right) /\left|x-\xi_{k}\right|\right)} \frac{x_{1}-x_{1}^{(k)}}{\left|x-\xi_{k}\right|}\right]\right\} \\
& \cdot f(x) d x \\
& =\sum_{k=1}^{n}\left(\mathrm{~V} \cdot \mathrm{p} \cdot \frac{1}{\left|x-\xi_{k}\right|^{2}}\left[-\frac{\partial a_{k}\left(\left(x_{1}-x_{1}^{(k)}\right) /\left|x-\xi_{k}\right|,\left(x_{2}-x_{2}^{(k)}\right) /\left|x-\xi_{k}\right|\right)}{\partial\left(\left(x_{1}-x_{1}^{(k)}\right) /\left|x-\xi_{k}\right|\right)} \frac{x_{2}-x_{2}^{(k)}}{\left|x-\xi_{k}\right|}+\frac{\partial a_{k}\left(\left(x_{1}-x_{1}^{(k)}\right) /\left|x-\xi_{k}\right|,\left(x_{2}-x_{2}^{(k)}\right) /\left|x-\xi_{k}\right|\right)}{\partial\left(\left(x_{2}-x_{2}^{(k)}\right) /\left|x-\xi_{k}\right|\right)} \frac{x_{1}-x_{1}^{(k)}}{\left|x-\xi_{k}\right|}\right],\right.
\end{aligned}
$$

$$
f(x)) \text {. }
$$

Thus, the following equality is true in the sense of generalized functions:

$$
\begin{aligned}
\operatorname{div} A & =\sum_{k=1}^{n} \mathrm{~V} \cdot \mathrm{p} \cdot\left\{\frac { 1 } { | x - \xi _ { k } | ^ { 2 } } \left[-\frac{\partial a_{k}\left(\left(x_{1}-x_{1}^{(k)}\right) /\left|x-\xi_{k}\right|,\left(x_{2}-x_{2}^{(k)}\right) /\left|x-\xi_{k}\right|\right)}{\partial\left(\left(x_{1}-x_{1}^{(k)}\right) /\left|x-\xi_{k}\right|\right)} \frac{x_{2}-x_{2}^{(k)}}{\left|x-\xi_{k}\right|}\right.\right. \\
+ & \left.\left.\frac{\partial a_{k}\left(\left(x_{1}-x_{1}^{(k)}\right) /\left|x-\xi_{k}\right|,\left(x_{2}-x_{2}^{(k)}\right) /\left|x-\xi_{k}\right|\right)}{\partial\left(\left(x_{2}-x_{2}^{(k)}\right) /\left|x-\xi_{k}\right|\right)} \frac{x_{1}-x_{1}^{(k)}}{\left|x-\xi_{k}\right|}\right]\right\} .
\end{aligned}
$$

The theorem is proved.

Screening every thin solenoid $\widetilde{\xi}_{k}=\left(x_{1}^{(k)}, x_{2}^{(k)}, x_{3}\right)(k=$ $\left.1,2, \ldots, n, x_{3} \in R_{1}\right)$ with the use of Dirac function $\delta(x-$ $\left.\xi_{k}\right)(k=1,2, \ldots, n)$, we obtain a multicenter Schrödinger operator

$$
\begin{aligned}
& (i \nabla+A(x))^{2}-b_{1} \delta\left(x-\xi_{1}\right)-b_{2} \delta\left(x-\xi_{2}\right)-\cdots \\
& \quad-b_{n} \delta\left(x-\xi_{n}\right)
\end{aligned}
$$

with the magnetic Aharonov-Bohm potential of type (1), where $b_{k}$ 's $(k=1,2, \ldots, n)$ are real numbers.

Consider in $L_{2}\left(R_{2}\right)$ the symmetric operator $H_{0}$ with the domain $D\left(H_{0}\right)=C_{0}^{\infty}\left(R_{2} \backslash\left\{\xi_{1}, \xi_{2}, \ldots, \xi_{n}\right\}\right)\left(C_{0}^{\infty}\left(\Omega^{\prime}\right)\right.$ is the totality of all infinitely differentiable finite functions in $\Omega^{\prime}$ ), which acts as follows:

$$
\begin{aligned}
H_{0} \psi(x)=(i \nabla+A(x))^{2} \psi(x), & \\
& \psi(x) \in C_{0}^{\infty}\left(R_{2} \backslash\left\{\xi_{1}, \xi_{2}, \ldots, \xi_{n}\right\}\right) .
\end{aligned}
$$


We denote by $H$ the closure of the operator $H_{0}$. Let

$$
\int_{-\pi}^{\pi} a_{k}(\theta) d \theta=\tilde{a}_{k}+\alpha_{k}, \quad k=1,2, \ldots, n,
$$

where $\widetilde{a}_{k}$ is the integral part and $\alpha_{k}$ is the fractional part of the number $\int_{-\pi}^{\pi} a_{k}(\theta) d \theta$. Obviously, $0 \leq \alpha_{k}<1, k=1,2, \ldots, n$. Without loss of generality, we will assume that there exists an integer $l \leq n$ such that

$$
\begin{gathered}
0<\alpha_{j}<1, \quad \text { if } j=1,2, \ldots, l, \\
\alpha_{j}=0, \quad \text { if } j=l+1, l+2, \ldots, n .
\end{gathered}
$$

Theorem 4. (i) The domain $D\left(H_{0}^{*}\right)$ of the conjugate operator $H_{0}^{*}$ coincides with the set

$$
\begin{aligned}
& D\left(H_{0}^{*}\right)=\left\{\psi(x): \psi(x) \in L_{2}\left(R_{2}\right)\right. \\
& \left.\quad \cap W_{2, \text { loc }}^{2}\left(\Omega^{\prime}\right), \quad(i \nabla+A(x))^{2} \psi(x) \in L_{2}\left(R_{2}\right)\right\},
\end{aligned}
$$

where $W_{2, \text { loc }}^{2}\left(\Omega^{\prime}\right)$ is a local second-order Sobolev space.

(ii) Deficiency index of the operator $H$ is $(2 l, 2 l)$, where $l$ is an integer $(l \leq n)$ defined in (40).

Proof. (i) As the domain of the operator $H_{0}$ is dense in $L_{2}\left(R_{2}\right)$, it has a conjugate operator $H_{0}^{*}$. The domain of this conjugate operator $D\left(H_{0}^{*}\right)$ is the totality of all $\psi(x)$ from $L_{2}\left(R_{2}\right)$ for which there exist $u(x) \in L_{2}\left(R_{2}\right)$ such that

$$
\left(H_{0} \varphi(x), \psi(x)\right)=(\varphi(x), u(x)) \text {, }
$$

for every $\varphi(x) \in D\left(H_{0}\right)$, and $H_{0} \psi(x)=u(x)$. From

$$
\left(H_{0} \varphi(x), \psi(x)\right)=\left(\varphi(x), H_{0}^{*} \psi(x)\right),
$$

it follows that $u(x)=(i \nabla+A(x))^{2} \psi(x)$ in the sense of generalized functions in $C_{0}^{\infty}\left(\Omega^{\prime}\right)$. Hence, in view of the ellipticity of the operator $(i \nabla+A(x))^{2}$, we have $\psi(x) \epsilon$ $W_{2, \text { loc }}^{2}\left(\Omega^{\prime}\right)$ (see [6]).

(ii) Considering the notations

$$
\begin{aligned}
& A_{1}(x)=\sum_{k=1}^{l} a_{k}\left(\frac{x-\xi_{k}}{\left|x-\xi_{k}\right|}\right) \\
& \cdot \frac{1}{\left|x-\xi_{k}\right|^{2}}\left(-x_{2}+x_{2}^{(k)}, x_{1}-x_{1}^{(k)}\right), \\
& A_{2}(x)=\sum_{k=l+1}^{n} a_{k}\left(\frac{x-\xi_{k}}{\left|x-\xi_{k}\right|}\right) \quad x=\left(x_{1}, x_{2}\right) \in \Omega^{\prime}, \\
& \cdot \frac{1}{\left|x-\xi_{k}\right|^{2}}\left(-x_{2}+x_{2}^{(k)}, x_{1}-x_{1}^{(k)}\right), \\
& \quad x=\left(x_{1}, x_{2}\right) \in \Omega^{\prime},
\end{aligned}
$$

in (1), we rewrite the potential $A(x)$ in the form of the sum of two summands:

$$
A(x)=A_{1}(x)+A_{2}(x)
$$

Now we introduce the magnetic $l$-flux potential

$$
\begin{array}{r}
B(x)=\sum_{k=1}^{l} \frac{\alpha_{k}}{\left|x-\xi_{k}\right|^{2}}\left(-x_{2}+x_{2}^{(k)}, x_{1}-x_{1}^{(k)}\right), \\
x=\left(x_{1}, x_{2}\right) \in \Omega^{\prime},
\end{array}
$$

where $\alpha_{k}$ is the fractional part of the number $\int_{-\pi}^{\pi} a_{k}(\theta) d \theta$.

It is proved in [7] that the minimal operator $H_{0, B}$ generated by the differential expression $(i \nabla+B(x))^{2}$ has the deficiency index $(2 l, 2 l)$. It follows from the results of $[7,8]$ that $A_{1}(x) \sim B(x)$ and $A_{2}(x) \sim 0$; that is, the pairs of potentials $\left(A_{1}(x), B(x)\right)$ and $\left(A_{2}(x), 0\right)$ are gauge equivalent. Consequently, the assertion (ii) of the theorem follows from the gauge equivalence of the potentials $A(x)$ and $B(x)$. The theorem is proved.

Remark 5. The assertions of Theorem 4 stay true if the Aharonov-Bohm solenoids lie in a homogeneous magnetic field of intensity $\gamma$, that is, for potentials of the form

$$
A(x)+\left(-\frac{\gamma}{2} x_{2}, \frac{\gamma}{2} x_{1}\right)
$$

Now let us make a few concluding remarks about the mathematical justification for the $\mathrm{AB}$ effect. Proceeding from Berezin and Faddeev's idea (see [9]), we arrive at the conclusion that the rigorous mathematical justification for the Aharonov-Bohm effect is that the pure Aharonov-Bohm operator $H_{\mathrm{AB}}$ lies among the self-adjoint extensions of the operator $H_{0}$; that is,

$$
H_{0} \subset H_{\mathrm{AB}} \subset H_{0}^{*} \text {. }
$$

For local and nonlocal $\delta$-interactions without magnetic field this idea was confirmed in many works (see, e.g., [10-13]), while for the Aharonov-Bohm operator it was confirmed in $[7,8,14]$. So the following question remains open for the potential of form (1): which of the self-adjoint extensions of the operator $H_{0}$ corresponds to the pure Aharonov-Bohm operator $H_{\mathrm{AB}}$ ?

\section{Conflict of Interests}

The authors declare that there is no conflict of interests regarding the publication of this paper.

\section{Acknowledgments}

This work was supported by the Deanship of Scientific Research (DSR), King Abdulaziz University, Jeddah, under Grant no. 377-1435. The authors, therefore, gratefully acknowledge the DSR technical and financial support. 


\section{References}

[1] Y. Aharonov and D. Bohm, "Significance of electromagnetic potentials in the quantum theory," Physical Review Letters, vol. 115, pp. 485-491, 1959.

[2] S. N. M. Ruijsenaars, "The Aharonov-Bohm effect and scattering theory," Annals of Physics, vol. 146, no. 1, pp. 1-34, 1983.

[3] G. N. Afanasiev, "Old and new problems in the theory of the Aharonov-Bohm effect," Physics of Elementary Particles and Atomic Nuclei, vol. 21, no. 1, pp. 172-250, 1990 (Russian).

[4] B. Alziary, J. Fleckinger-Pellé, and P. Takáč, "Eigenfunctions and Hardy inequalities for a magnetic Schrödinger operator in $\mathbb{R}^{2}$," Mathematical Methods in the Applied Sciences, vol. 26, no. 13, pp. 1093-1136, 2003.

[5] G. N. Afanasiev, "On the single-valuedness of wave functions in multiply connected spaces," JINR Rapid Communications, vol. 6, pp. 17-26, 1985 (Russian).

[6] S. Agmon, Lectures on Elliptic Boundary Value Problems, vol. 2 of Van Nostrand Mathematical Studies, Van Nostrand, Princeton, NJ, USA, Prepared for Publication by B. F. Jones Jr. with the Assistance of G. W. Batten Jr., 1965.

[7] T. Mine, "The Aharonov-Bohm solenoids in a constant magnetic field," Annales Henri Poincaré, vol. 6, no. 1, pp. 125-154, 2005.

[8] Y. Nambu, “The Aharonov-Bohm problem revisited," Nuclear Physics B, vol. 579, no. 3, pp. 590-616, 2000.

[9] F. A. Berezin and L. D. Faddeev, "Remark on the Schrödinger equation with singular potential," Doklady Akademii Nauk SSSR, vol. 137, pp. 1011-1014, 1961 (Russian).

[10] S. Albeverio, F. Gesztesy, R. Høegh-Krohn, and H. Holden, Solvable Models in Quantum Mechanics, Springer, Berlin, Germany; Mir Publishers, Moscow, Russia, 1988.

[11] E. H. Eyvazov, "Expansion in decisions scattering problem for the operator $H_{\alpha}+q(x)$," Doklady Akademii Nauk Azerbaĭdzhana, vol. 33, no. 10, pp. 8-11, 1977 (Russian).

[12] E. H. Eyvazov, "Higher order differential operators with finite number of $\delta$-interactions in multivariate case," Journal of Mathematical Physics, Analysis, Geometry, vol. 6, no. 3, pp. 266276, 2010.

[13] L. Dabrowski and H. Grosse, "On nonlocal point interactions in one, two, and three dimensions," Journal of Mathematical Physics, vol. 26, no. 11, pp. 2777-2780, 1985.

[14] L. Dabrowski and P. Stovcek, "Aharonov-Bohm effect with delta type interaction," Journal of Mathematical Physics, vol. 39, pp. 47-62, 1998. 


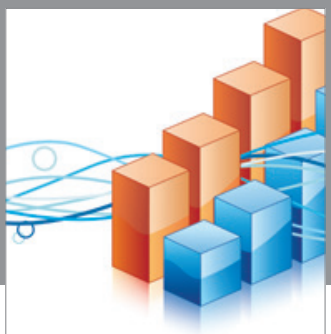

Advances in

Operations Research

vatem alat4

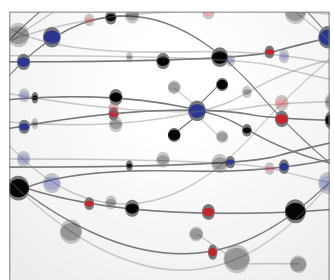

\section{The Scientific} World Journal
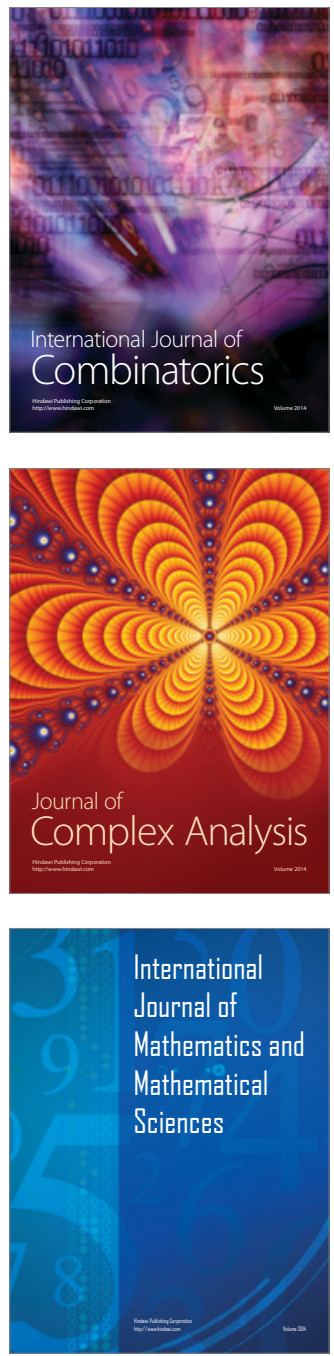
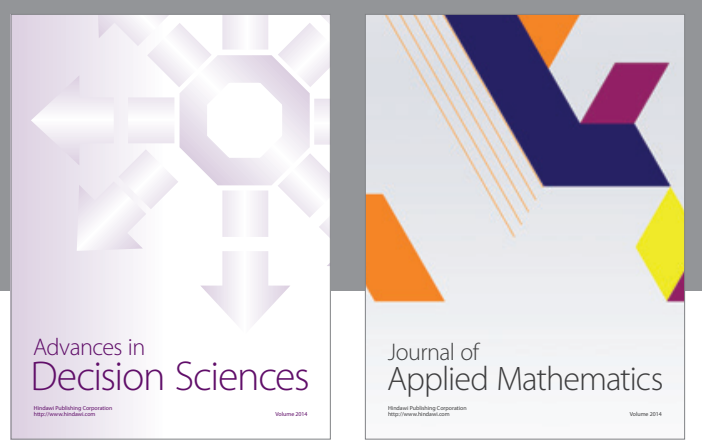

Algebra

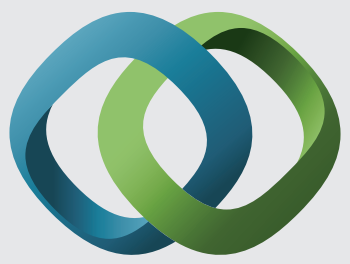

\section{Hindawi}

Submit your manuscripts at

http://www.hindawi.com
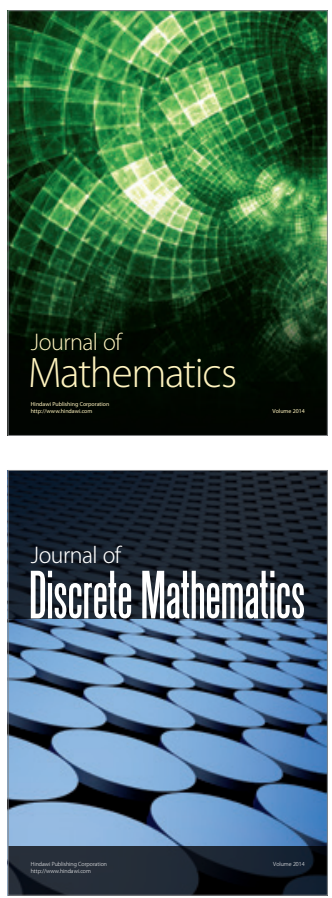

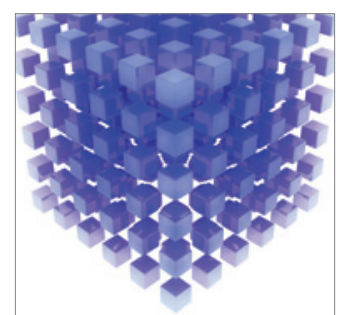

Mathematical Problems in Engineering
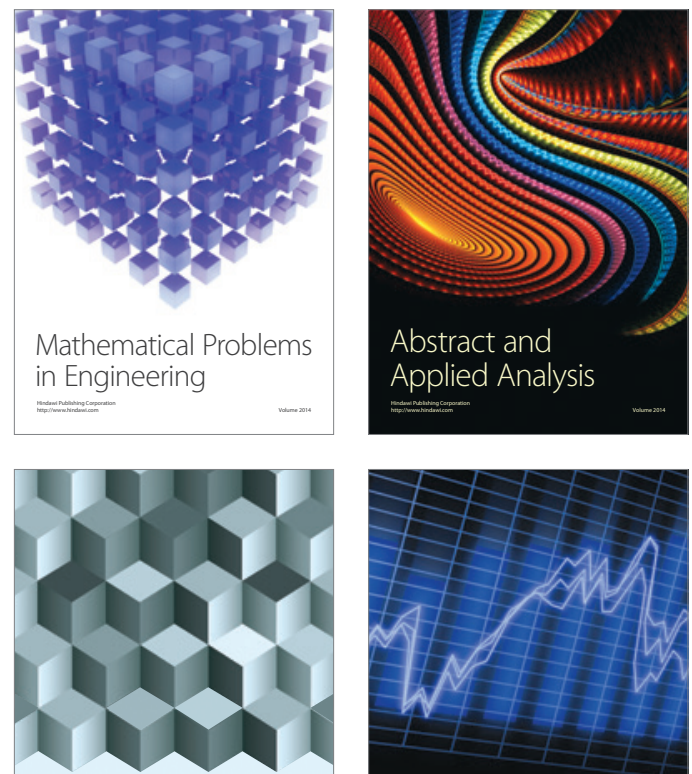

Journal of

Function Spaces

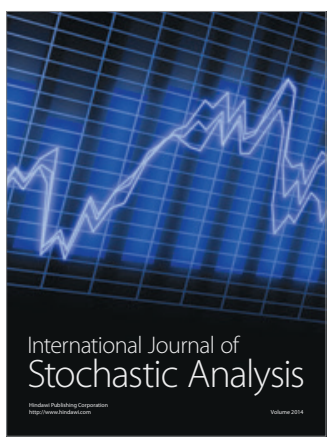

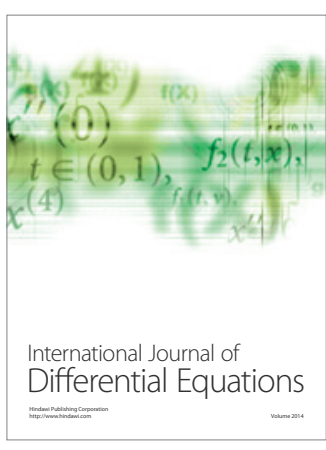
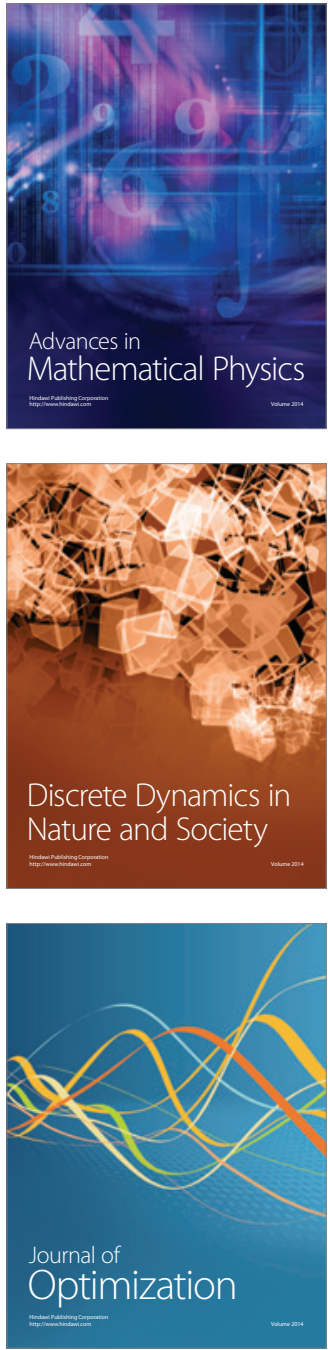